\title{
Implementing BPMN 2.0 Scenarios for AAL@Home Solution
}

\author{
L. Rusu, B. Cramariuc, D. Benta, M. Mailat
}

\section{Lucia Rusu}

Babeş Bolyai University of Cluj-Napoca

Romania, 400591 Cluj-Napoca, M. Kogălniceanu, 1

lucia.rusu@econ.ubbcluj.ro

\section{Bogdan Cramariuc}

IT Center for Science and Technology Bucharest

Romania, 011702 Bucharest, Av. Radu Beller, 25, sector 1

bogdan.cramariuc@ieee.org

Dan Benţa*

Agora University of Oradea

Romania, 410526 Oradea, Piaţa Tineretului, 8

*Corresponding author: dan.benta@univagora.ro

\section{Marinela Mailat}

Babeş Bolyai University of Cluj-Napoca

Romania, 400591 Cluj-Napoca, M. Kogălniceanu, 1

marinelamailat@yahoo.com

\begin{abstract}
Aging tendency of European population and live longer and independently desire requires AAL solution for particular elders (chronic diseases, disabilities, aso). NITICS project aim is to develop advanced ITC solutions including monitoring and navigational support for indoor to support elderly in their daily activities. This paper offers a BPMN implementation for indoor assistance based on IoT (sensor monitoring) and Activity workflow implementation. Our solution offers an intelligent Care Center solution for caregivers monitoring and elders support.
\end{abstract}

Keywords: IoT, AAL@Home, BPA, BPM, BPMN, workflow.

\section{Introduction}

The European population is aging and tends to live longer and independently. This requires for a good quality of life staying at home. European Commission (EC) Ambient Assisted Living Joint Program (AAL JP) offers an opportunity to incorporate the technological progress in communications, Internet-of-Things (IoT) and Artificial Intelligence (AI). Moreover, advances in medical and assistance or caring sciences are increasingly making people with disabilities autonomous and self-sufficient. Advanced ICT services including monitoring and navigational support are needed to support the mobility of elderly and disabled persons in their home during their daily activities [1].

IoT has many definitions, focused on infrastructure, object interconnection, context awareness, communication interoperability, security, privacy or other particular features. IoT means "things having identities and virtual personalities operating in smart spaces using intelligent interfaces to connect and communicate within social, environmental, and user contexts" [2]. SAP definition offered by Stephan Haller, SAP AG, focuses on business processes "a world where physical objects are seamlessly integrated into the information network and where the physical objects can become active participants in business processes". Services are available to interact with these 'smart objects' over the Internet, query and change their state and any information associated with them, taking into account security and privacy issues. 
Based on IoT features for addressing elements such as Convergence, Content, Collections (Repositories), Computing, Communication, and Connectivity from the context, this concept could be applied for homecare solutions which needs interconnection between people and things. Ambient Assisted Living (AAL) focused on several areas AAL4persons: AAL@Home, AALonthe-move, AAL@Work and AAL@Community.

Other authors talk about AAL as home care systems (HCS) which are focused on the support of living assistance for people with special needs (elderly, disabled) in their own homes. HCS domain can be divided into three parts: emergency treatment services, autonomy enhancement services, and comfort services. Emergency Treatment covers assistance, detection, prediction, prevention services. These services offer early prediction and recovery from critical conditions, emergency and safe detection and alert propagation of emergencies (sudden falls, heart attacks, strokes, panics, aso) .Autonomy Enhancement is focused on eating, drinking, dress, medication, cooking, cleaning, shopping services. Comfort Services cover all areas that do not fall into the previous categories: logistic, finding things, home automation, social contacts, infotainment, safety [3].

This paper offers a solution for AAL@Home using SmartHome concept as an Assisted Living Technologies (ALT), by offering a BPMN 2.0 implementation which helps to assist elder people. After an introduction, in the second section we deal with business process analysis (objectives and specific features, actors and roles in our project), and then we develop several workflow scenarios using AAL@Home features, based on end-user (elderly and care-givers) requirements, which include ALT and Telecare features. Last section presents conclusions and future work.

\section{Related results}

There were previously mentioned some AAL solutions for particular elders.

ROBOCARE, is an Italian AAL@Home research project focuses on the development of distributed systems with software and robotic agents for generating active services in environments for humans may need assistance and guidance. This solution allowing vulnerable elderly people to lead an independent lifestyle in their own homes. ROBOCARE has two scenarios: the ROBOCARE Domestic Environment (RDE) and the Health-Care Institution (HCI) scenario. It covers several fields of research: robotic platforms, sensory systems, activity supervision in complex environments, and human-technology interaction [4].

ROBOCARE investigate the integration of robotic, sensory and automated reasoning components into RDE and HCI scenarios. This project offers main aspects related to technology for elderly care: development of the enabling domotic components (intelligent sensors and robotic platforms) for deployment the target scenarios and development functionalities of domotic components (activity supervision and diagnostics) using service-providing software infrastructure [4].

Smart-home architecture was described by Bregman and Korman using interaction of four modules: Central Management Units (CMU), User Interface (UI), Home Equipment and Appliances Interface (HEAI), External Communication Interface (ECI). The CMU has several components: Operating System (SHOS - Smart Home Operating System), the Smart-Home Database (SHDB), AI (Artificial Intelligence) Engine or Home Intelligence (HI) and Application Services (AS) [5]. An implementation of this model was done in www.liorzehome.com using objectoriented approach. Each event is defined as a set of properties: Event Name: a description of the event name, Input Devices with devices inside the eHome that trigger an event, and functions: Event Triggers: that return TRUE when a trigger is invoked, Output Operation with the desired output operation, Output Alert, the eHome Main Alerts defines the UI for main alerts. If an event requires an alert, Output Alert describes the alert. The design follows Object-Process Methodology (OPM), OPCAD, OPDs (Object-Process diagrams) [6]. 
Another solution for Ambient Intelligence based home care systems (AHCS) was offered by amiCA (AmI Care and Assistance) prototype for monitoring Monitoring drinking, Monitoring Food Quality, Location Tracking and Fall detection, using different amiCA services interconnected by service platform which was developed in BelAmI project (Bilateral German-Hungarian Collaboration Project on Ambient Intelligence System), based on SOA paradigm. COAALAS (COmpanion for Ambient Assisted Living on Alive-ShareIt platforms) aim is to model the sensor network around disabled users as societies, with the expected behavioral patterns, for supporting smart assistive tools [7]. From a huge HCS we mention AALOA (AAL Open Association) which is supported by several AAL projects: BRAID (FP7), MonAMI (FP6), OASIS (FP7), OsAmI-Commons (ITEA2), PERSONA (FP6), SOPRANO (FP6), universAAL (FP7) and WASP (FP6) [8].

\section{NITICS Business Process Analyze (BPA)}

NITICS Project within the AAL Program (Network Infrastructure for Innovative home Care Solutions) aim is to develop an advanced ICT solution which includes monitoring and navigational support for indoor and to support elderly people in their daily activities (nutrition, personal hygiene, home care) in the context in which an integrated solution is still missing [9].

Main Operational objectives are [1]:

- a) Defining and designing a flexible service platform allowing and facilitating the integration and consolidation of existing elements, leading to a continuous improvement and extension of end-user services. NITICS also provides opportunities to design new customized services to better take care about the end-user;

- b) Improving the quality of life of elderly and disabled persons by allowing them to be mobile in a safe way inside the house and sustain them in their daily life activities;

- c) Improving self-sufficiency of elderly and disabled persons, by self-caring at home (selfcheck of health conditions and life-style, medication reminder, nutrition status monitoring and alerting), in order to avoid excessive workload and cost from the involved caregivers

- d) Improving the response in terms of efficiency (quality and speed) from the care providers and from the individual's family in emergency situations, by an alarming system, by sensors/cameras feedback to carers, by remotely controlling devices and by video conversations with carers.

Providing care in an efficient way, by making use of reliable information on the condition of the elderly, which will indicate whether an informal carer is needed for aid or if a more specialized formal carer has to intervene.

In our project we have identified four categories of actors: end-users, caregivers, IT specialists, application and service providers. End-users are elderly and people with diseases and disabilities.

Because of agile methodology for system development application and service providers should be able to develop and implement applications and offer maintenance and upgrade services, according to end-users or caregivers demand.

First step in our system development was to analyze the end-user requirements. We have applied two questionnaires for elderly people (over 65 years) in order to identify the main activities in which they need help and also to identify the type of help needed. After first questionnaire we have identified two scenarios to address the needs of the users: 
- activities that require physical help - in which case a platform is needed that triggers a request for help either automatically (e.g. vital parameters are out of range, falling alarm, etc) or through the interaction with the user (e.g. button pressing). The request is sent to a caregiver which can be a professional, family, neighbor or friend (AAL@home features).

- needs that can be addressed fully by an ITC platform alone: reminders, day organizers, virtual entertainment and interaction, etc (AAL@home and AAL@Comunity features).

Based on our results, we re-define Siciliano classifications for NITICS project, ordering need items by importance, as obtained from the interviews and we update challenges and electronic support according to end-users requirements. In hierarchical classification we used three clusters: needs, support and challenges and we obtained connections between these clusters linked with basic needs for elders living [1].

Second questionnaire has 61 items (Q1-Q61), and was applied on 59 persons, elder people between 65 and 83 years, with chronically or severe diseases, several of them (12\%) have also disabilities. End-users hierarchically needs and electronically support was classified hierachicaly from very important need (first row) to less important need (last row). Another needs classification is focused on caregivers help to solve their needs. Needs that require help from a caregiver prevail and needs that can be addressed without direct help form caregiver.

We focused on first category of needs for the third survey regarding the caregiver perspective on the elderly needs and their perception towards various dimensions of daily life (i.e., health, interaction with technology, health care etc.). The interviews were based on a questionnaire specifically developed for this purpose which comprised 32 items (Q1-Q32) divided in seven sections: demographic data and other primary measures, specificity of caregiving services, the client related impact, evaluation the opinion of the caregivers, according to their own experience, on the importance of the functionalities of an ICT platform towards facilitating the delivery of caregiving services to the elderly, technology acceptance and the users' perception in relation with the project goals (interest in future participation in surveys and system testing).

\section{NITICS Business Activity Monitoring (BAM)}

NITICS has developed using RDE and HCI scenarios detailed by Cesta and Pecora based on several domotic components and functionalities of domotic components using service-providing software infrastructure [4]. This section offers only HCI scenarios for Emergency Treatment of elders with special needs and implementation was developed based on agile methodology, using domotic components, SOA and intelligent agents. If we consider a private Care Center that can be assimilated as an organization, which has its own Business Process (BP). Care Center activity can be a Business Process, which is defined as a collection of related tasks that produce a specific service or product (to serve a particular goal, in our case elders care) for a particular customer: elderly people [10]. BPMN 2.0 specification defines three models for different aspects of processes: Process Model, Choreography Model and Collaboration Model. To achieve the planned objectives of the project, of the center as an organization we use the Process Model that describes how operations are carried out [11].

Because our target was a care center like an entity, we model the process at a private level of abstraction, as an internal Business Processes. In future work-package we shall develop public levels as collaborative B2B Processes.

We used the software Activiti 5.13 as a platform for BPMN 2.0, released under the Apache open source, written in Java, which can run in any Java application, on a server, in the cluster or in the cloud. It is an alternative implementation of BPMN 2.0 jBPM (JBoss BPM). Activiti 
Modeler offers a solution modeling analyst BPMN 2.0 business process that can be compiled in a web browser. This process can be easily distributed because it requires no client software before one starts modeling. Activiti Designer is an Eclipse plug-in (editing application framework) that allows developers to improve its process modeling activity in a process Modeler BPMN 2.0, which can be executed by the processing engine. Activiti Explorer provides an overview of the processes implemented, management, interacting with them and viewing tables in the database managed processing engine [12].

The control of this Care Center is done through a web portal in which access is allowed to both health care professionals, patients, and their relatives. Recording and monitoring equipment in patients' rooms uses XMPP server, which are scripts that monitor and take the equipment alarms and sending the data to the interpretation workflows PubSub method (Publish and Subscribe). Workflows are managed by Active framework, which runs each process. Sending data is ensured by BOSH transport protocol and for identifying each user equipment and network, Jabber employs a unique identifier (JID), consisting of the user name @ name of the server that houses the respective users. Equipment registered is required to be monitored by assigning a JID unique for each.

Portal scripts require monitoring a particular device. When it receives an alarm from one of the sites, it creates a device notification and follows the chain of tasks assigned for each type of alarm process diagrams, from active to complete execution of that process. Scripts are responsible for announcing persons assigned a specific task through the portal by notification by SMS or email. Client control section presents the apartment of a patient with all the equipment found in him. In the living room are the following sensors:

- Main Entrance Access Point: represented by the apartment alarm system, it indicates that the system is armed or not;

- Entrance sensor: a sensor that transmits if the apartment front door is opened or closed;

- Living room siren: it is a siren that can start in case of serious alarms;

- Window Sensor: a sensor that transmits if the window is open or not;

- Living room heating: a sensor attached to a radiator that indicates whether heater is switched into the chamber;

- Living room thermostat: A thermostat that can show several indicators, such as whether the heater is switched on, room temperature or desired temperature.

In the bedroom are some of the sensors that are in the room

- Presence Bedroom: a motion sensor that indicates whether someone is in the room or when he or she has moved last time.

- Bedroom light: indicates whether the bedroom light is on / off, and brightness level.

- Monitoring Camera: camera that monitors the patient's room.

- Bedroom Alarm Button: is a button next to the bedside alarm that can be pressed in an emergency.

- Bedroom Floor sensor: in the floor in front of the bed there is a sensor to track whether this is down from the bed and cannot get up. 


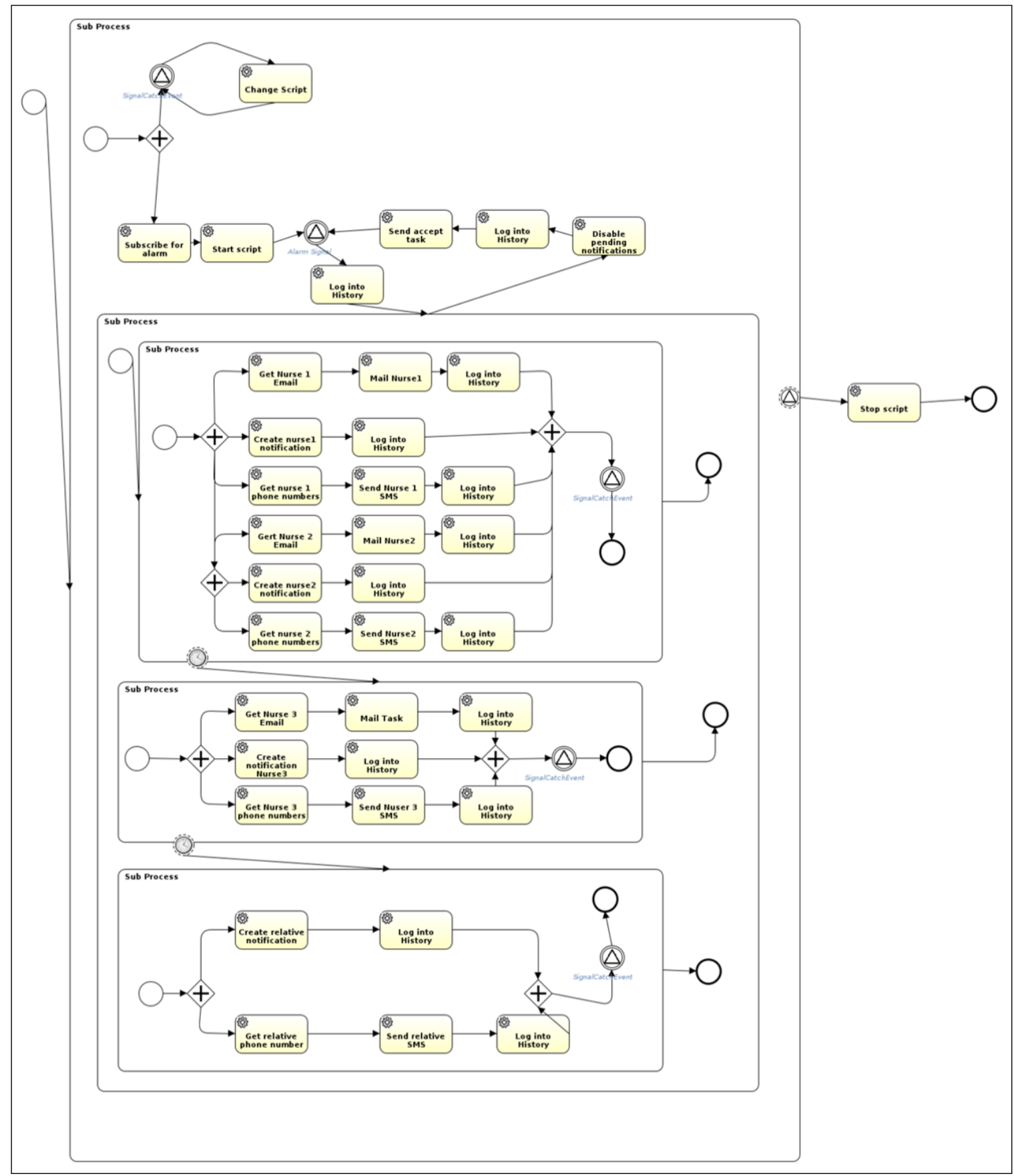

Figure 1: BPMN implementation for Bed Alarm workflow

- Bed sensor (Bed occupancy sensor) indicates whether the bed is occupied or not, specifically whether or not it is the patient bed.

In addition, there are other sensors as required for patient safety: Presence Bedroom, Bedroom light, Monitoring Camera, Bedroom Alarm Button, Bedroom Floor sensor, Bed occupancy sensor. All sensors have the option to get the history of the events that have triggered, and the ability to perform certain tasks directly accessing the portal, without having displacement chamber. These tasks can be reinforcement safety system, turning heat, light switch, changing luminosity, opening and closing the door or window. 
We exemplify BPMN implementation for Bed Alarm workflow (Fig. 1). Equipment that governs the workflow is the sensor bed - Bed Occupancy Sensor. This sensor triggers an alarm if the patient exceeds a certain number of hours without getting up from bed.

For example, if the patient does not rise over 10 hours suggesting that the patient may have an ailment. From here the patient is monitored Bed Alarm workflow is started by setting specific parameters. PubSub technique requires scripting to monitor the equipment. From the moment the script start monitoring waiting sensor bed alarms. When an alarm is received, it is recorded in history, and then starts a sub alarm to those who need to take alarm and to execute the task.

Depending on the parameters set to start workflow are determined people who will receive this alert. For the default parameters in parallel performs the following tasks: obtain assistance one email address, it sends an e-mail that is announced by this alarm, it creates a portal notification for this nurse to obtain his phone number and to send an SMS to this notice. At the same time it is announced and nurse 2, following the previous steps (similar to nurse 1). Once these tasks have been completed, it sends a signal that the first two nurses were announced and the first sub-ending. After the time set to start workflow, if neither of the two nurses did not take the alarm then goes ahead and announces the following persons.

Patient's relatives are notified only by SMS and notification portal, and the response time is greater than of the nurses. When one of them takes the alarm notification, then the script is disabled. The historical record sends permission to perform the task, and the script goes back to the state of waiting for a new alarm monitoring. Demonstration parameters that can start this workflow are the following: o Maximum occupancy: 8:00 p.m. o Alarm Priority: high o Type of alarm : SMS, email and notification portal o The receiver alarm 1: nurse 1 and nurse 2 if they do not take the alarm in 5 minutes; o Alarm receiver 2: two nurse ( who has already been notified by the portal, but not via email or SMS) unless you take alarm in 5 minutes, o Alarm receiver 3: relative 1 and relative 2 (will be notified through the portal and SMS), if those do not accept within 30 minutes; o Alarm receiver 4: audible alarm emergency room.

\section{Conclusions and future works}

Our solution offers a BPM approach for an intelligent home care solution. Elderly care in a specialized institution may be considered a business process. The existence of repetitive tasks enables automation of these processes. Each process is modeled and it is presented as a set of individual activities or by compound several sub-processes. The modeling process shows the activities in the order in which they occur, the actors performing the activities, inputs and outputs for each activity, information flows input/output during the process, the rules used in the process. All these are made with the Activiti framework, which may possess all components to develop complex workflows. BPMN 2.0 standard underpinning this framework and it facilitates the work of business analysts and developers, providing the right set of graphics required modeling process. Process automation is a necessity because it allows labor efficiency for nurses and specialized caregivers. Indeed, they are not forced to make regular visits to patients, just only when the visits are needed. In addition the health, elderly people can be easily traced. The proposed model is safe for patients being monitored continuously and it also ensures that their relatives or friends are well cared for. By comparison with other existent solution, we focused on process automation and modeling workflows using IoT elements.

\section{Acknowledgment}

The work presented has been founded by the research Grant FP7 AAL Joint Programme, Networked InfrasTructure for Innovative home Care Solutions, and was financed by Center IT 
for Science and Technology, Romania.

\section{Bibliography}

[1] NITICS (Network Infrastructure for Innovative home Care Solutions) (2013), AAL Joint Program - (C) NITICS (4 of 33) Call AAL-2012-5, http://pl.ssw.org.pl/portofolioview/dolore-magna-aliqua

[2] Botterman, M., (2009); Internet of Things: an early reality of the Future Internet. Workshop Report, European Commission Information Society and Media.

[3] Nehmer, J., Karshmer, A., Becker, M., Lamm, R.( 2006); Living Assistance Systems - An Ambient Intelligence Approach. Proceedings of the International Conference on Software Engineering (ICSE), DOI: 10.1145/1134285.1134293, 43-50.

[4] Cesta, A., Pecora, F., (2005); The ROBOCARE Project: Intelligent Systems for Elder Care, American Association for Artificial Intelligence, www.aaai.org

[5] Bregman D.; Korman, C. (2009); A Universal Implementation Model for the Smart Home, International Journal of Smart Home, 3(3): 15-30.

[6] Zoref, L.; Bregman, D.; Dori, D. (2009); Networking Mobile Devices and Computers in an Intelligent Home, International Journal of Smart Home, 3(4): 15-22..

[7] Becker, M.; Werkman, E.; Anastasopoulos, M.; Kleinberger, T. (2007); Approaching Ambient Intelligent Home Care Systems, Pervasive Health Conference and Workshops, 2006, DOI: 10.1109/PCTHEALTH.2006.361656, 1-10.

[8] Universal open platform and Reference Specification for Ambient Assisted Living, http://www.universaal.org/index.php/en

[9] Serbanati, A.; Medaglia, C.M.; Ceipidor, U.B. (2011); emphBuilding Blocks of the Internet of Things: State of the Art and Beyond, ISBN 978-953-307-380-4, 382 pages, InTech Publisher.

[10] White, S.A.; Miers, D. (2008); BPMN Modeling and Reference Guide: Understanding and Using BPMN, Future Strategies Inc., Lighthouse Point, Florida, USA.

[11] OMG, 'Business Process Model and Notation (BPMN): Version 2.0 specification', Technical Report formal/2011-01-03, Object Management Group, (January 2011).

[12] http://www.activiti.org/userguide/, last access: 12.07.2013. 